\title{
The Limits and Extension of Transformation Optics
}

\author{
Martin McCall ${ }^{*}$ and Paul Kinsler ${ }^{\dagger \dagger}$ \\ * Department of Physics, Imperial College London, UK \\ e-mail: m.mccall@imperial.ac.uk \\ $\dagger^{\dagger}$ Cockcroft Institute, Keckwick Ln, Daresbury WA4 4AD, U.K. \\ ${ }^{\ddagger}$ Physics Department, Lancaster University, Lancaster LA1 4YB, U.K.
}

\begin{abstract}
We explore an apparent limitation of transformation optics, namely that a given transformation induces polarizationdependent impedance gradients that might lead to scattering. This observation must be reconciled with the idea that the transformation optics algorithm is 'exact', and leads to perfect morphing of the electromagnetic field without inducing scattering. We also discuss the role of curvature in transformation optics, showing that the conventional algorithm is nothing other than an interesting re-representation of flat Cartesian (Minkowskian) space (spacetime), without any curvature. However, we also consider possible extensions to transformation optics, to schemes that embrace curvature, and include so-called anholonomic transformations. We also show that the conventional spatial transformation optics algorithm can locally be described by six numbers.
\end{abstract}

\section{INTRODUCTION}

Transformation optics is a design recipe that permits, within fairly mild constraints, the actualization of the morphing of the electromagnetic field in an essentially arbitrary way. The most celebrated example is of course the electromagnetic cloak, in which a region of space is excised by surrounding it with a suitable metamaterial design. Light is curved around the region so that observers are unaware of any objects that the region contains. [1]-[4].

There are a number of interesting and fundamental issues provoked by Transformation Optics that will be discussed in this paper:

1) Despite its exactness, Transformation Optics presents an intriguing puzzle. We will show that using TO it is easy to construct media for which impedance gradients and impedance anisotropies are present. These will presumably induce scattering, destroying the perfection of a given design. Through some simple pedagogical examples, we will show how this paradox is resolved.

2) What role, if any, does (or can) curvature play in transformation optics? It is not widely appreciated, for example, that the conventional algorithm, being a diffeomorphism of Euclidean space, does not, by construction, induce any curvature. However, our recent studies suggest that curvature can play a role in certain schemes.

3) Can transformation optics be related to 'simple' optics, determined by optical parameters such as the refractive at a point in an arbitrary direction? The fact that transformational media are necessarily anisotropic suggests that this will not be possible, as two indices will in general be required for a given direction of propagation. However, the nature of the transformation optics algorithm is such that in fact for any direction of propagation there is only one refractive index, independent of polarization [5]. This opens up the possibility that the transformation optics algorithm can be interpreted, at least locally, in a simple and intuitive geometrical way.

\section{The Impedance PuZzle}

It is very easy to construct a simple example of transformation optics that induces a medium with non-trivial impedance properties. Consider the trivial morphism that dilates one spatial direction

$$
(\tilde{x}, \tilde{y}, \tilde{z})=(x, y, \lambda z),
$$

where $\lambda$ is a dilation factor. Transformation optics then dictates that the resulting constitutive tensor $\epsilon=\boldsymbol{\mu} \equiv \tilde{\boldsymbol{\kappa}}$ is given by

$$
\tilde{\boldsymbol{\kappa}}=\operatorname{diag}\left(\lambda^{-1}, \lambda^{-1}, \lambda\right) .
$$

We find that for such a medium the unique refractive index seen by a ray propagating in the $x-z$ plane at angle $\theta$ to the $z$-axis is given by

$$
n(\theta)=\left(\lambda^{2} \cos ^{2} \theta+\sin ^{2} \theta\right)^{1 / 2} .
$$

Along the direction of dilation $(\theta=0) n(0)=\lambda$, and orthogonal to this direction $(\theta=\pi / 2)$ the vacuum value $n(\pi / 2)=1$ is maintained. However, despite the fact that $\boldsymbol{\epsilon}=\boldsymbol{\mu}$, the impedance behaviour of the medium is in general polarization dependent. For a field propagating along the $z$-direction, the impedance is independent of polarization and equal to its vacuum value $\eta_{0}=\left(\mu_{0} / \epsilon_{0}\right)^{1 / 2}$, but for a field propagating along the $x$-direction we find that the field components satisfy

$$
\left(\begin{array}{c}
E_{y} \\
E_{z}
\end{array}\right)=\eta_{0}\left(\begin{array}{cc}
0 & \lambda \\
-\lambda^{-1} & 0
\end{array}\right)\left(\begin{array}{l}
H_{y} \\
H_{z}
\end{array}\right) .
$$

If the electric field points along $y$ the impedance is $\lambda$ times its vacuum value, whilst if the field points along $z$, the impedance is $\lambda^{-1}$ times its vacuum value. Surely such impedance anisotropy must be commonplace (in fact inevitable) in any transformation optics scheme? Such considerations led us, in a recent paper [6], to suggest that 'perfect' cloaking is impossible. However, more recently we have sought to reconcile Transformation-induced impedance with the idea that transformation optics is 'exact', i.e. that a given morphism 
can, in principle, be replicated exactly by an appropriate metamedium, without scattering. We will present a simple example to resolve the paradox. Moreover, the example we present can be used to achieve 'perfect refraction', i.e. a medium that responds as though light from vacuum incident at any angle and carrying any polarization will be yield a Brewster-like zero-reflection at the vacuum-medium interface.

\section{The Role of CuRvature in Transformation OPTICS}

Consider a diffeomorphism of some abstract space, $\varphi$ : $\mathcal{M} \rightarrow \mathcal{M}$ having a local coordinate representation $x^{\tilde{\mu}}=$ $x^{\tilde{\mu}}\left(x^{\nu}\right)$, that can represent the morphing of space (or spacetime) to be implemented via transformation optics. The diffeomorphism induces a set of connection coefficients $\tilde{\Gamma}_{\mu \nu}^{\rho}$ related to those present before the morphism is applied $\left(\Gamma_{\beta \gamma}^{\alpha}\right)$, given by

$$
\tilde{\Gamma}_{\mu \nu}^{\rho}=\left(\varphi_{*}\right)_{\alpha}^{\tilde{\rho}}\left(\varphi^{*}\right)_{\tilde{\mu}}^{\beta}\left(\varphi^{*}\right)_{\tilde{\nu}}^{\gamma} \Gamma_{\beta \gamma}^{\alpha}+\left(\varphi^{*}\right)_{\tilde{\mu}, \tilde{\nu}}^{\alpha}\left(\varphi_{*}\right)_{\alpha}^{\tilde{\rho}},
$$

where where $\left(\varphi_{*}\right)_{\alpha}^{\tilde{\rho}}=\partial \tilde{x}^{\rho} / \partial x^{\alpha}$ and $\left(\varphi^{*}\right)_{\tilde{\mu}}^{\beta}=\partial x^{\beta} / \partial \tilde{x}^{\mu}$ The induced connection coefficients set a new standard of parallelism by which geodesics can be compared. A simple example is the famous cloaking transformation illustrated in Fig. 1 and defined by

$$
r^{\prime}=\left(1-\frac{a}{b}\right) r+a, \quad \theta^{\prime}=\theta, \quad z^{\prime}=z,
$$

where $a$ and $b$ are positive constants with $b>a$. The

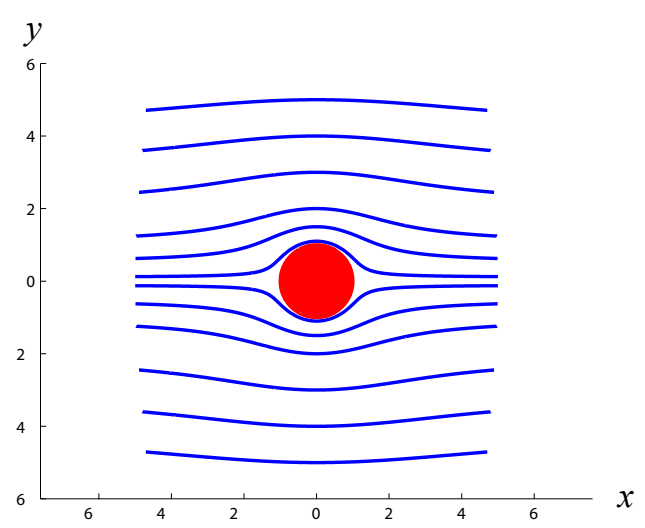

Fig. 1. The coordinate transformation of Eq. (6) for the lines of constan $y=r \sin \theta$.

'curved' trajectories induced by a cloaking transformation of parallel light rays are actually preserved to be 'straight' under the morphism, and the standard of straightness set by Eq. (5). Furthermore, an explicit calculation of the curvature tensor using the induced connection coefficients of (5) reveals this to be everywhere zero. Explicity, the induced curvature components are given by

$$
\tilde{R}_{\mu \nu \lambda}^{\rho}=\left(\varphi_{*}\right)_{\alpha}^{\tilde{\rho}}\left(\varphi^{*}\right)_{\tilde{\mu}}^{\beta}\left(\varphi^{*}\right)_{\tilde{\nu}}^{\gamma}\left(\varphi^{*}\right)_{\tilde{\lambda}}^{\delta} R_{\beta \gamma \delta}^{\alpha} .
$$

Hence, by construction, if $\mathcal{M}$ is flat, no diffeomorphism can make it curved. Referring again to the example of Fig. 1 we can say that the induced curves are actually preserved to be parallel. In short, curves do not make curvature [7]!

However, as we will explain in the presentation, there is scope for embracing true curvature within Transformation Optics once the type of allowed transformation is extended beyond diffeomorphisms, or where the base manifold $\mathcal{M}$ is curved anyway. These can be related in a simple and natural way to recent demonstrations that illustrate gravitational lensing in guided wave optical systems [8].

\section{JUST SIX Numbers}

The electromagnetic medium $\boldsymbol{\epsilon}=\boldsymbol{\mu}$ defined by any spatial diffeomorphism has the coordinate representation

$$
\tilde{\kappa}^{l m}=\left|\operatorname{det}\left(\frac{\partial \tilde{x}^{i}}{\partial x^{j}}\right)\right|^{-1} \frac{\partial \tilde{x}^{l}}{\partial x^{i}} \frac{\partial \tilde{x}^{m}}{\partial x^{j}} \kappa_{0}^{i j},
$$

where in Cartesians $\kappa_{0}^{i j}=\delta^{i j}$. We showed recently ( [6]) how such a medium is completely described by a refractive index $n(\mathbf{r}, \hat{\mathbf{s}})$ that depends on position $\mathbf{r}$ and direction $\hat{\mathbf{s}}$, but is independent of polarization. As a result of this degeneracy the phase surface is always ellipsoidal, with the ellipsoid's three principal axes defining three principal indices $\left(n_{1}, n_{2}, n_{3}\right)$, with the ellipsoid orientation being described by three angles $(\Phi, \Theta, \Psi)$. These six local quantities completely describe the optical properties of the medium and if known can be used to reconstruct the diffeomorphism that gave rise to the medium. Explicitly, the refractive index at a point $\mathbf{r}$ in direction $\mathbf{s}$ is given (in any orthonormal basis) by

$$
n=\left[\operatorname{det}\left(\boldsymbol{\kappa}^{-1}\right)_{\perp}\right]^{-1 / 2},
$$

where $\perp$ represents the projection into the plane orthogonal to s. Although the construction is simple and elegant, it would be nice to generalize it beyond orthonormal bases, as will be discussed in the presentation.

\section{ACKNOWLEDGMENT}

Useful discussions with Robert Thompson and Paul Kinsler are acknowledged.

\section{REFERENCES}

[1] J. B. Pendry, D. Schurig, and D. R. Smith, "Controlling electromagnetic fields," Science, vol. 312, pp. 1780-1782, 2006

[2] U. Leonhardt and T. G. Philbin, "General relativity in electrical engineering," New Journal of Physics, vol. 8, no. 247, 2006.

[3] U. Leonhardt, "Optical conformal mapping," Science, vol. 312, pp. $1777-$ $1780,2006$.

[4] D. Schurig, J. J. Mock, B. J. Justice, S. A. Cummer, J. B. Pendry, A. F Starr, and D. R. Smith, "Metamaterial electromagnetic cloak at microwave frequencies," Science, vol. 314, pp. 977-980, 2006.

[5] A. Favaro and L. Bergamin, "The non-birefringent limit of all linear, skewonless media and its unique light-cone structure," Ann. Phys. (Berlin), vol. 523, p. 383, 2011.

[6] M. W. McCall, P. Kinsler, and R. D. M. Topf, "The refractive index of reciprocal electromagnetic media," Accepted for Journal of Optics.

[7] P. Kinsler and M. W. McCall, "The futures of transformations and metamaterials," Photonics and Nanostructures, vol. 15, pp. 10-23, 2015.

[8] C. Sheng, H. Liu, Y. Wang, S. N. Zhu, and D. A. Genov, "Trapping light by mimicking gravitational lensing," Nature Photonics, vol. 7, pp. 902-906, 2013 